\title{
Broadening of the Iron emission line in MCG-6-30-15 by Comptonization
}

\author{
R. Misra \\ A. K. Kembhavi \\ Inter-University Centre for Astronomy and Astrophysics, Pune, India
}

Received — 


\begin{abstract}
We show that the Iron K emission line from MCG-6-30-15 could be broadened due to Comptonization by a surrounding highly ionized cloud with radius $\sim 10^{14}$ cms. We calculate the temperature of the cloud to be $\sim 0.21 \mathrm{keV}$, provided a reasonable estimate of the UV flux is made. The X-ray/ $\gamma$-ray emission observed from the source is compatible with this model. Such a cloud should be highly ionized and strong absorption edges are not expected from the source (Fabian et al. 1995).

For a $10^{6} M_{\odot}$ black hole the size of the could corresponds to about 300 Schwarzschild radius. The intrinsic line could then be emitted far from the black hole and gravitational red-shift and Doppler effects would be negligible. If the black hole mass is much larger than $10^{6} M_{\odot}$, gravitational/Doppler red-shifts would also contribute significantly to the broadening.

We argue that the broad red wing observed in the source does not by itself imply emission from regions close $\left(R<5 r_{s}\right)$ to the black hole. However, Comptonization cannot produce a double peak. The presence of such a feature is a clear sign of inner disk emission influenced by gravitational and Doppler effects, perhaps broadened by the Comptonization. We note that simultaneous broad band (2-100 keV) study of this source can also reveal (or rule out) the presence of such a Comptonizing cloud.
\end{abstract}

Subject headings: accretion disks-black hole physics - galaxies:individual (MCG-6-30-15) — galaxies:Seyfert_-line:profile 


\section{Introduction}

Recent ASCA observations have shown that the Iron $\mathrm{K}$ emission lines in the spectra of many Seyfert 1 galaxies are broad (Tanaka et al. 1995; Mushotzky et al. 1995). A long $(\approx 4.5$ days) observation of the bright Seyfert 1 galaxy MCG-6-30-15 showed that observed line width corresponds to a velocity $\sim 10^{10} \mathrm{~cm} \mathrm{~s}^{-1}$ and is asymmetric with most of the emission being red-shifted (Tanaka et al. 1995). Furthermore, no strong absorption edges were observed from this source. It was realized that such emission is expected when the line is produced in the inner region $3 r_{s}<R<10 r_{s}$ about a black hole, where $r_{s}=2 G M / c^{2}$ is the Schwarzschild radius. The line shape is due to the combined effect of gravitational and Doppler red-shifts ( Fabian et al. 1989 ). Spectral fitting revealed that the line is variable and that during one epoch ( the deep minimum phase ) it seems to be generated close $\left(\approx 2 r_{s}\right)$ to a spinning (Kerr) black hole (Iwasawa et al. 1996). If this interpretation is true this would be the first direct observation of the strong gravitational effects expected close to a black hole. Moreover, this result strongly constrains theoretical models. Since the cold accretion disk extends all the way to the last stable orbit, models having a hot inner disk (for e.g. Shapiro, Lightman \& Eardley 1976; Misra \& Melia 1996; Narayan \& Yi 1994; Chakrabarti 1997) would no longer be valid for this source. At the same time the X-ray source would need to be situated very close to the black hole $\left(\approx 2 r_{s}\right)$. Models where the X-rays are produced in hot active regions (or coronas) ( for e.g. Liang \& Price 1977; Haardt \& Maraschi 1993) above the cold disk would be more promising. However, at least for MCG -6-30-15, these active regions would now have to be concentrated near the last stable orbit instead of an inner region $\left(\approx 20 R_{s}\right)$ as has been hitherto assumed.

The importance of these results and their interpretation warranted the examination of alternative models which could explain the observations. Fabian et al. (1995) considered several other kinds of mechanisms for the broadening and concluded that none of them 
was satisfactory, thus strengthening the case for the inner disk emission model. One of the possibilities considered by Fabian et al. (1995) was the broadening of a narrow intrinsic line by Comptonization (Czerny, Zbyszewska and Raine 1991). They concluded that to explain the line profile the electron temperature in the Comptonizing medium would have to be less than $0.25 \mathrm{keV}$ and have an optical depth $\tau=5$. Moreover, in order not to have large photo-electric edges, the region was required to be highly ionized. Fabian et al. (1995) found this model unsatisfactory because (a) for the medium to be highly ionized the region should be less than $50 r_{s}$ from a $10^{7} M_{\odot}$ black hole, which would make gravitational effects dominant, (b) the X-ray emission would be altered by such a covering medium, and c) the Compton temperature of this region is expected to be higher than $0.25 \mathrm{keV}$, which is inconsistent with the upper limit on the temperature obtained from the profile.

In this paper we argue that the present $\mathrm{X}$-ray $/ \gamma$-ray observations of this source are compatible with a covering Comptonizing cloud. We calculate the self consistent temperature by balancing input to the output power in this cloud and find that the temperature is $\approx 0.2 \mathrm{keV}$, provided a reasonable estimate of the UV flux in this source is made. We show that the essential features of the line, namely, a broad red wing and a sharp blue drop can also be reproduced by a Comptonized line spectrum. Thus, it may be possible that a Comptonizing cloud with radius $\approx 10^{14} \mathrm{cms}$ surrounding the black hole contributes significantly to the broadening of the Iron line. In this case, the innermost region of an accretion disk $\left(r<10 r_{s}\right)$ could still be hot, with the reflection arising from the inner optically thick disk $\left(10 r_{s}<r<30 r_{s}\right)$. The active regions in the hot corona models may then be uniformly situated over the inner region of an optically thick disk $\left(r<20 r_{s}\right)$ where most of the gravitational energy is dissipated. However, the presence of a Comptonizing medium would raise questions regarding it's dynamical support, stability, geometry and origin. 


\section{The Cloud Model}

We consider a uniform cloud of radius $R$ surrounding the black hole with scattering optical depth, $\tau_{s c} \approx 5$. For no absorption edges to be observed the gas has to be highly ionized. Following Fabian et al. 1995 we first consider the outer layers of the optically thick cloud. The outer layer here is defined such that $\tau=n_{e} \sigma_{T} \Delta R \approx 1$, where $n_{e}$ is the electron density of the cloud and $\Delta R$ is the thickness of the outer layer. Kallman \& McCray (1982) have calculated the ionization equilibrium for such clouds provided $\tau_{s c}<0.3$. Since in this layer the scattering optical depth is less than unity, their calculations are approximately valid. Furthermore since multiple scattering is rare in this region, their calculation for the equilibrium temperature would also be approximately correct. Their results indicate that the outer layer of the cloud is highly ionized if the ionization parameter $\Xi \equiv L / n_{e} R^{2}>10^{4}$, where $L \approx 10^{43} \mathrm{ergs} \mathrm{sec}^{-1}$ is the luminosity of the source. Thus the maximum radius for this cloud is $R<10^{14} \mathrm{cms}$ ( Fabian et al. 1995). Iwasawa et al. 1996 report that the line is variable on a time scale less than $10^{4} \mathrm{sec}$, which also implies that the size of the cloud is $R<3 \times 10^{14} \mathrm{cms}$. For a $10^{7} M_{\odot}$ black hole, this would correspond to $\approx 30 r_{s}$. We note that this upper limit on the radius (relative to $r_{s}$ ) of the Comptonizing cloud depends on the assumed mass of the black hole. For example, if the black hole mass is $10^{6} M_{\odot}$, the limit would be $\approx 300 r_{s}$. For these conditions, Kallman \& McCray (1982)

calculate the equilibrium temperature to be $\approx 1 \mathrm{keV}$. This temperature is higher than the temperature implied from the line profile (Fabian et al. 1995). However, this is the outer layer temperature. The temperature of the inner regions of the cloud (where most of the Comptonization occurs) is expected to be less than this value, due to multiple scattering ( see below).

Estimating the ionization state of the gas in the inner region of the cloud is complicated, due to multiple scatterings of the photons. The calculations of Kallman \& McCray (1982) 
are no longer valid. Since this region is closer to the X-ray source the photon flux is higher here; moreover the photon density inside is also expected to be $\tau$ times higher than the value calculated using the free streaming approximation. From these arguments it is expected that the cloud should be more ionized in the interior than the outer layer. On the other hand the equilibrium temperature in the inner region is expected to be smaller due to multiple scatterings.

From the above arguments, Fabian et al. (1995) concluded that a cloud with radius $R>10^{14} \mathrm{cms}$, will not be highly ionized. This upper limit on the size is compatible with the observed variability of the source. Thus if a Comptonizing cloud surrounds this source its radius should be less than $10^{14} \mathrm{cms}$.

\section{The effects of Comptonization}

In this paper we consider the effect of a uniform Comptonizing cloud with $R=10^{14}$ cms on the X-ray continuum and line shape. As an extreme case, we neglect gravitational and Doppler broadening, which would be justified if the black hole mass is $\approx 10^{6} M_{\odot}$. The spectrum of the central source before the Comptonization is assumed to be an X-ray power-law with an exponential cutoff, a UV bump and a reflected component. The UV photons are most probably the intrinsic emission of the reflecting surface. Hence, for consistency, the UV photon source is also assumed to be within the Comptonizing cloud. The output spectrum after the Comptonization is obtained by solving the Kompane'ets equation with this spectrum as the source. We take into account bremsstrahlung photon production and absorption in the cloud. However, we find that neither of these is important for the parameters chosen here. The distance to the source is taken to be $50 \mathrm{Mpc}$ (red-shift $z=0.008$ with $\left.H_{0}=50 \mathrm{~km} \mathrm{sec}^{-1} \mathrm{Mpc}^{-1}\right)$. 
The temperature of the cloud was calculated by balancing the input radiative power to the output power. For this purpose, the Kompane'ets equation was solved by assuming some value for the temperature. Then the luminosity in the output (i.e., Comptonized) spectrum was compared to the intrinsic luminosity of the central source. The temperature was then varied till the luminosities were equal. This equilibrium temperature is a function of the scattering optical depth $\tau$ in the cloud and the estimated UV flux of the source.

In Figure 1, the dashed line shows the intrinsic spectrum assumed by us. The dotted line is the best fit X-ray spectrum of MCG-6-30-15 as observed by Ginga (Nandra \& pounds 1994, Table 8, Set 3), in the $2-18 \mathrm{keV}$ range. Based on this we take the power-law photon index of the spectrum to be 2.07 . We assume that the spectrum is exponentially cutoff at $E_{c}=600 \mathrm{keV}$ and that the reflection parameter $R_{\text {ref }} \equiv \Omega / 2 \pi=1.2$. The UV bump is assumed to have a nearly black-body like shape,

$$
F_{u v}=A E^{0.3} \exp \left(-E / E_{T}\right) \text { photons } / \mathrm{sec} / \mathrm{cm}^{2} / \mathrm{keV} .
$$

Here $E$ is the photon energy and $E_{T}$ is assumed to be $20 \mathrm{eV}$. The form is chosen to match with the average UV spectral slope of 1.3 observed in Seyferts (Kinney et al. 1991). The UV flux at $1550 \AA$, i.e., $8 \mathrm{eV}$ is observed to be correlated to the X-ray flux at $2 \mathrm{keV}$ ( Gondhalekar, Rouillon-Foley \& Kellett 1996). We have estimated the UV flux using the analytical expression fitted to this correlation. The dot in Figure 1 represents this UV flux $\left(\approx 5 \times 10^{-26} \mathrm{ergs} \mathrm{s}^{-1} \mathrm{~cm}^{-2} \mathrm{~Hz}^{-1}\right)$ for the X-ray flux $\left(\approx 5 \times 10^{-29} \operatorname{ergs~s}^{-1} \mathrm{~cm}^{-2} \mathrm{~Hz}^{-1}\right)$ observed by Ginga.

The temperatures obtained for different optical depths, obtained using the input spectrum assumed for this source, are shown in Table 1. The temperature also depends on the estimated UV flux of the source.

The solid line in Figure 1 is the Comptonized spectrum from a cloud with $\tau=3$ and the calculated temperature of $0.22 \mathrm{keV}$. As shown below the system seems to be in this 
state for most of its time. In the energy band $2-18 \mathrm{keV}$ the spectrum is nearly equal to the intrinsic spectrum. Thus in the Ginga band the cloud does not produce any observable signatures. At $100 \mathrm{keV}$ the flux is reduced by about $60 \%$. The averaged Seyfert 1 spectrum from OSSE has been presented by Gondek et al. (1996). The flux times energy at $100 \mathrm{keV}$ lies between 0.02 and $0.03 \mathrm{keV} \mathrm{s}^{-1} \mathrm{~cm}^{-2}$, which is just compatible with the predicted value here. However, comparing with an averaged spectrum may be misleading since not all the Seyferts used in the sample have a broad Iron line and hence in the framework of this model do not have optically thick clouds. Moreover, the OSSE and ASCA observations are not simultaneous. The line width is variable which in this framework implies that the optical depth of the cloud also varies. Thus the Comptonized X-ray spectrum of MCG-6-30-15 is not in conflict with the present observations.

However, simultaneous broad band (2 - $200 \mathrm{keV})$ observations of this source would be able to test the cloud model. In particular, a break in the spectrum ( see figure 1) around $50 \mathrm{keV}$ would indicate the presence of a Comptonizing cloud. On the other hand if such a break is not observed and simultaneous data shows that the line is very broad, that would argue against a Comptonizing cloud. Thus simultaneous observations of this source by ASCA and RXTE or BeppoSAX may be able to test the Comptonizing cloud model. It should be noted that a significant break at $30 \mathrm{keV}$, has not been observed in any Seyferts so far (Gondek et al. 1996).

Table 1: Temperatures for different values of optical depth.

\begin{tabular}{|c|c|}
\hline \hline Optical Depth & Temperature \\
\hline 5 & 0.18 \\
3 & 0.22 \\
1 & 0.23 \\
\hline
\end{tabular}


We assume that the intrinsic Iron line is a Gaussian with dispersion $\sigma_{I}=0.2 \mathrm{keV}$. In figure 2 (a) we show this intrinsic line (dotted) and the Comptonized line (solid). Here $\tau=5$ and the temperature is $0.18 \mathrm{keV}$. The dashed line is the best fit double Gaussian model to the data obtained by Ginga for one set of observations by Iwasawa et al. (1996). This was named as the deep minimum phase and showed the maximum broadening. Although the Comptonized spectrum does not compare well with the best fit spectrum, some of the salient features are present: namely, there is an extended red wing and a sharp drop towards the blue side. In fact, comparison of the Comptonized spectrum with the unfolded photon spectrum shown in Figure 7 of Iwasawa et al. (1996), rather than with their best fit, reduces the discrepancy. In Figure $2(\mathrm{~b}), \tau=3$ and the temperature is $0.22 \mathrm{keV}$. The Comptonized spectrum is compared with the best fit double Gaussian fit obtained for the Intermediate flux state (Iwasawa et al 1996). The system was in this state for five of the seven sets of observations. Figure 2 (c) shows the Comptonized spectrum when $\tau=1$ and temperature is $0.23 \mathrm{keV}$ compared with the bright flare phase.

We note that the broadened spectrum due to Comptonization is not similar to the double peaked spectrum expected from inner disk emission. Higher resolution observations of the line would be able to differentiate between the two scenarios. In particular, if the double peaked nature of the line is observed (not just the broadening), then the emission would indeed be produced close to a spinning black hole. On the other hand, a single broadened line would be consistent with emission from a Comptonizing medium.

Spectral fitting of the X-ray band for MCG-6-30-15 requires the presence of a variable warm absorber with hydrogen column density $N_{H} \sim 6 \times 10^{22} \mathrm{~cm}^{-2}$. In the simplistic model presented here we have considered a uniform cloud with a definite radius. In reality, the density of the cloud probably decreases with radius and the low density region of the cloud extends to greater radii than $10^{14} \mathrm{cms}$ considered here. We speculate that the warm 
absorber could have its origin in this extended region of the cloud.

\section{Discussion}

In this paper, we show that an optically thick Comptonizing cloud with radius $R \approx 10^{14}$ cms, could contribute significantly to the broadening of an intrinsic iron line. The gas would probably be highly ionized and hence no absorption edges should be observed (Fabian et al. 1995). We find that taking into account the UV radiation from the source and multiple scattering in the medium, the equilibrium temperature of the cloud is $k T \approx 0.2 \mathrm{keV}$. The shape of the Comptonized line from such a medium is similar to the one observed. If the black hole mass is $\approx 10^{6} M_{\odot}$ the Compton broadening could be dominant. For a $10^{7} M_{\odot}$ black hole, gravitational/Doppler effects would also contribute.

The present hard X-rays observations are compatible with the spectrum expected from such a cloud. However, simultaneous broad band observation of this source (e.g. with ASCA, RXTE and BeppoSAX ) will be able to test the presence of such a Comptonizing cloud.

We emphasis that the spectral shape of the Comptonized line is different from the disk emission non-Comptonized one. In particular, the disk emission line is double peaked, while the Comptonized spectrum is continuous. If the line is observed to be double peaked, that would indicate that Comptonization is not dominant. However, direct evidence for the presence of a Comptonizing medium would be obtained, if the line is observed to be continuous. In such a scenario, the cold accretion disk need not extend to the last stable orbit, the black hole may not be spinning and the hard X-ray source need not be situated very close to the black hole. Thus, if there is a Comptonizing cloud, the X-ray producing regions could still be described by either an hot inner disk or a coronae (active region) on 
top of a cold disk. It is also possible that both Comptonization and gravitational red-shift contribute significantly to the broadening of the line.

The authors would like to thank A. Fabian for useful criticism of an earlier draft and A. A. Zdziarski for comments and discussion. 


\section{REFERENCES}

Chakrabarti, S.K., 1997, Astrophys. J. 484, 322.

Czerny B., Zbyszewska M. \& Raine, D.J. 1991, in Treves A., ed., Iron line Diagnostics in X-ray Sources, Springer-Verlag, Berlin, p 226.

Fabian, A.C., Rees, M.J., Stella, L. \& White, N.E. 1989, Mon. Not. Roy. astr. Soc. 238, 729.

Fabian, A.C. et al. 1995, Mon. Not. Roy. astr. Soc. 277, L11.

George, I.M. \& Fabian, A.C. 1991, Mon. Not. Roy. astr. Soc. 249, 352.

Gondhalekar, P.M., Rouillon-Foley, C. \& Kellett, B.J. 1996, Mon. Not. Roy. astr. Soc. $282,117$.

Gondek, D. et al. 1996, Mon. Not. Roy. astr. Soc. 282, 646.

Haardt, F. \& Maraschi, L., 1993, Astrophys. J. 413, 507.

Iwasawa, K. et al. 1996, Mon. Not. Roy. astr. Soc. 282, 1038.

Kallman, T.R. \& McCray, R. 1982, Astrophys. J. Suppl. 50, 263.

Kinney, A.L., Antonucci, R.R.J., Ward, M.J., Wilson, A.S. \& Whittle, M. 1991, Astrophys. J. 377, 100.

Liang, E.P. \& Price, R.H., 1977, Astrophys. J. 218, 247.

Misra, R. \& Melia, F., 1996, Astrophys. J. 467, 405.

Mushotzky, R.F., 1995, Mon. Not. Roy. astr. Soc. 272, p9.

Narayan, R. \& Yi, L., 1994, ApJ428, L13.

Shapiro, S.L., Lightman A.P. \& Eardley, D.M. 1976, Astrophys. J. 204, 187.

Nandra, K. \& Pounds, K.A. 1994, Mon. Not. Roy. astr. Soc. 268, 405. 
Tanaka, Y., 1995, Nature 375, 659. 
Fig. 1.- The intrinsic spectrum of the central source (dashed line) and the Comptonized spectrum from a cloud with $\tau=3$ (solid line). The spectral fit to Ginga data in the energy band 2-18 keV (dotted line) and the estimated UV flux (dot).

Fig. 2.- The intrinsic narrow Iron line from the central source (dotted line) and the Comptonized Iron line from a cloud (solid line). The best fit double Gaussian model fit to data (dashed line). (a) for the deep minimum phase compared to line from a cloud with $\tau=5.0$. (b) for the intermediate flux phase compared to line from a cloud with $\tau=3.0$. (c) for the high flare phase compared to line from a cloud with $\tau=1.0$. 


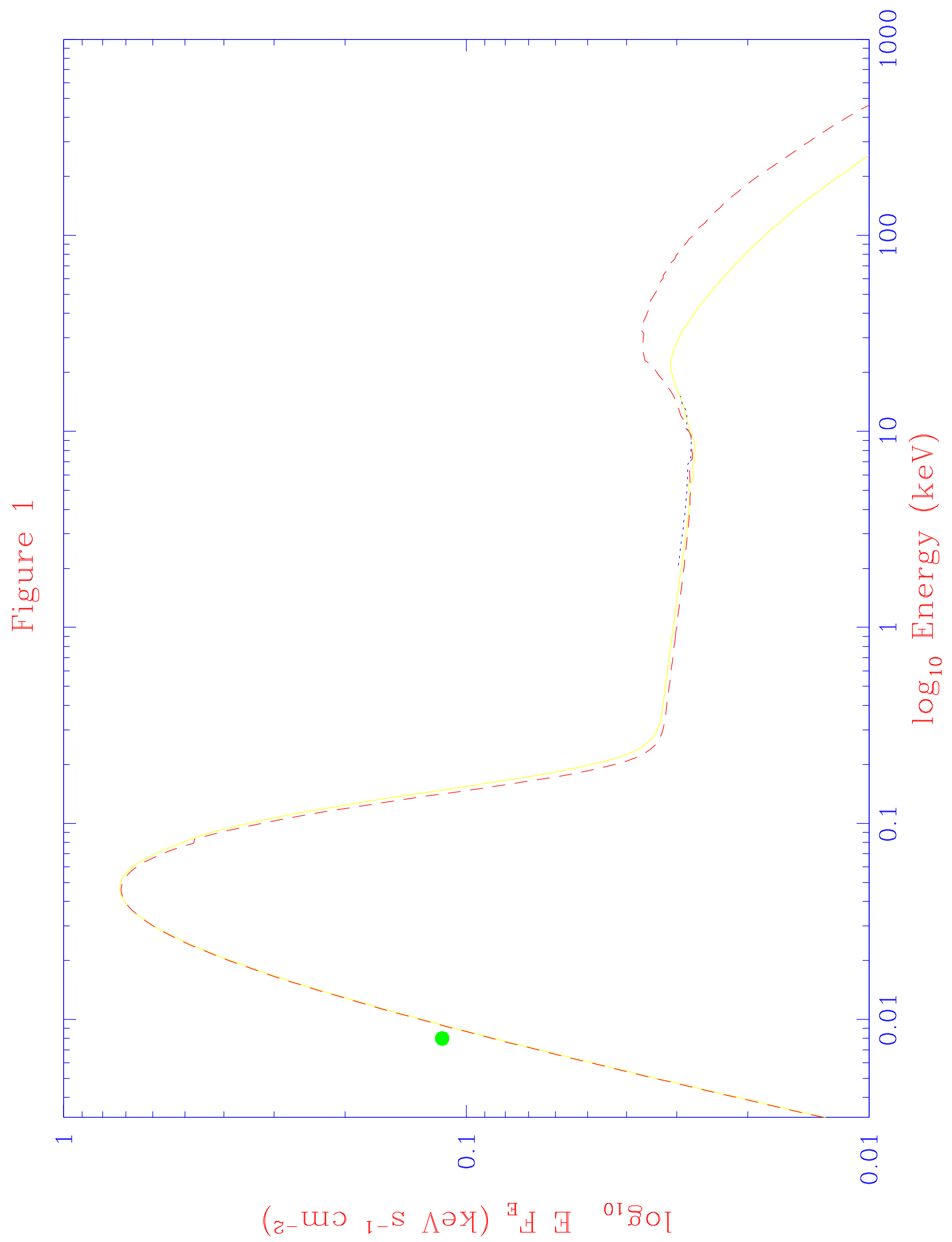


Figure 2

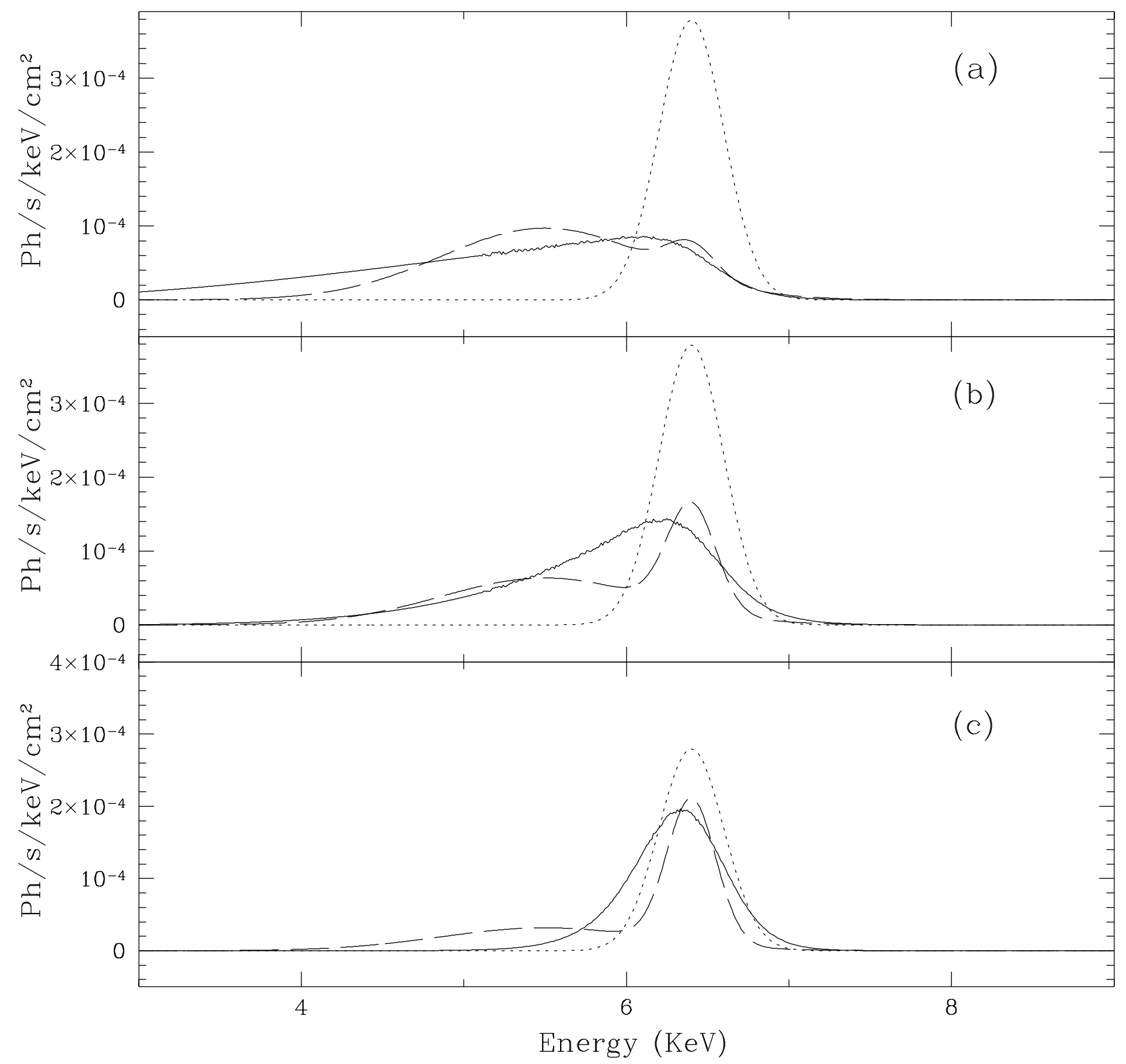

